\title{
FUNDUS REFLECTOMETRY, AN EXPERIMENTAL STUDY
}

\author{
CHAPTER I
}

\section{INTRODUCTION}

When considering the many questions that still arise concerning the haemodynamics of the posterior segment of the eye, distinction must be made between the retinal and choroidal vasculature. Although the retinal circulation is still to be investigated more intensively, there exists a fair amount of information on this item. Photographic methods (HICKAM \& FRAZER, 1966) make it possible to give fairly accurate estimates of the diameter of the larger retinal arteries and veins and their arteriovenous oxygen differences. The mean circulation time in the retinal vessels can be calculated (BULPITT \& DOLLERY, 1971) and so can alterations in this time under influence of a raised intra-ocular pressure (DOLLERY et al., 1968).

Not so for the circulation in the choroid. Covered by a layer of dense pigment, it is hardly accessible to fluorescein angiographic research. Also cardiogreen, which enables the use of longer wavelength light, which is absorbed to a lesser degree by the pigment epithelium, does not give satisfying information (BROWN \& STRONG, 1973).

Many research workers could not resist the temptation to tap vortex veins in animals (BILL, 1962a; 1962b; BEST et al., 1972; NAKAMURA \& GOULSTINE, 1973; SCHLEGEL \& LAWRENCE, 1969) to catheterize the long posterior ciliary artery (BILL, 1963a), to place probes (BILL, 1963b; NIESEL \& KONSTAS, 1959), or to dehydrate the sclera (GREAVES \& PERKINS, 1952) in order to measure flow, record pressures or study the behaviour of choroidal vessels under the microscope. Unfortunately all these methods are apt to disturb the physiological conditions of the eye.

Others used radioactive isotopes (BETTMAN \& FELLows, 1956, 1958a, 1958b, 
1962; PILKERTON, BULLE \& O'ROURKE, 1964; FRIEDMAN, 1970; FRIEDMAN \& CHANDRA, 1972; CHANDRA \& FRIEDMAN, 1972; ALM \& BILL, 1973a, 1973b; WEITER et al., 1973a, 1973b; STRANG, WILSON \& JOHNSON, 1974; WILSON et aI., 1973) in order to give estimates of choroidal bloodflow and volume. Continuous measurements are practically impossible even with these advanced techniques. The situation now is such that any contribution to the resolution of the problem is welcome as it might replace or complete earlier less reliable experiments. Most of the available data refer to the situation at one moment only. We thought it essential to study the circulatory relationships in the eye with a continuous measurement. Because disturbance of the physiology of the eye, by for example catheterizing vessels or applying detectors, may easily result in changed haemodynamics, it was thought essential as second condition not to touch the eye if possible.

Fundus reflectometry was chosen as the technique best answering to our purpose. This method, also named fundus densitometry or fundus spectophotometry, has been a fascinating method of examining certain aspects of the human and animal eye, since Rushton (1955) brought this technique to a high level of development for his work on visual pigments.

He designed a system of light-bundles of different colours which entered the eye through the dilated pupil. The amount of light returning was measured. BROADFOOT, GLOSTER \& GREAves (19.61) were the first to use alternating lightbundles of different wavelength ranges. They found, when measuring the intensity of different wavelengths of light that returned from the fundus, a change in these intensities under varying conditions of the experimental animal. They were able to demonstrate in albino rabbits and to a certain extent in humans (GLOSTER, 1967), the effect of a decreased oxygen saturation, of higher intra-ocular pressure and of some drugs. These authors made use of the changing relationship in the amount of $\mathrm{Hb}$ and $\mathrm{HbO}_{2}$ present in the vascular layers and of the fact that $\mathrm{Hb}$ and $\mathrm{HbO}_{2}$ have a different spectral absorption characteristic.

TROKEL $(1964,1965)$ described an elegant densitometric method to calculate the bloodflow in the choroid of albino rabbits by means of a dye-dilution technique. However, as at the end of each experiment dye was injected in the vitreous, each animal could serve for one experiment only.

Measurements of the blood layer thickness were done by PEREGRIN \& DODT (1969) in albino rabbits. They obtained highly reproducible results. Their method made use of subtracting the logarithm of the intensity of the returning light from that of the light returning from a bloodless eye. This one bloodless eye was considered to be the standard for all subsequent experiments. They 
were able to demonstrate the influence of pulsewave and respiration on the blood layer thickness of the choroid.

DE COCK elaborated a more modern technique (BAKKER, DE COCK et al., 1973), derived from that of RUSHTON \& BROADFOOT. He introduced fibre optics and a contact lens to provide greater versatility. Monochromatic light of a half intensity of $1,2 \mathrm{~nm}$, enabled the use of exactly the desired wavelength. Although reflectometry is not necessarily a superior method compared with other methods of examining the choroid, we felt reflectometry might give more information than previous investigations, just by using more precise techniques. The subject of this study was therefore to investigate whether rcliable continuous measurcments of qualitative and quantitative changes in blood layer thickness and blood oxygen saturation were possible, without disturbing the physiology of the eye.

Fundus reflectometry as elaborated by OOSTERHUIS (OOSTERHUIS, BAKKER \& VAN DEN BERGE, 1970; OOSIERHUIS \& BAKKER, 1973), where the fundus of the eye is used as a background against which the passage of dye can be registered, will not be dealt with. Rabbits with their merangiotic retinal vasculaturc (restricted to the area of medullated nerve fibres in the upper quadrant of the cye) are suitable experimental animals for investigation of the choroidal circulation. Since in pigmented rabbits a much lower percentage of the incident light returns from the fundus of the eye than in albino rabbits, the latter are preferable for most experiments.

In reporting the results of our investigations, we found it better, for readability's sake, to divide the material into chapters, each dealing with a limited part of the subject. As a consequence of this several concepts will be mentioned at a stage where they may still be unfamiliar to the reader. A glossary has been added to help overcome this difficulty. 